\title{
Binding Mode and Induced Fit Predictions for Prospective
}

\section{Computational Drug Design}

Christoph Grebner ${ }^{* 1}$, Jessica legre ${ }^{2}$, Johan Ulander ${ }^{1}$, Karl Edman $^{3}$, Anders Hogner $^{1}$,

Christian Tyrchan*2

${ }^{1} \mathrm{CVMD}$ Innovative Medicine, ${ }^{2} \mathrm{RIA}$ Innovative Medicine, ${ }^{3}$ Discovery Science, AstraZeneca

R\&D 43283 Mölndal, Sweden; *christoph.grebner@astrazeneca.com,

christian.tyrchan@astrazeneca.com

\section{Supporting Information}

\begin{tabular}{|l|c|}
\hline ANM/PCA modes for c-Src and Abl & $\mathrm{SI}$ \\
\hline ANM/PCA modes for ER and HNE & $\mathrm{SI}$ \\
\hline ANM/PCA modes for Syk & $\mathrm{SI}$ \\
\hline LRMSD and backbone RMSD from MD simulations: HNE & $\mathrm{SI}$ \\
\hline Covered phase space MD/PELE: HNE & $\mathrm{SI}$ \\
\hline LRMSD and backbone RMSD from MD simulations: ER (1U3Q) & $\mathrm{SI}$ \\
\hline Covered phase space MD/PELE: ER (1U3Q) & $\mathrm{SI}$ \\
\hline LRMSD and backbone RMSD from MD simulations: ER (1U3S) & $\mathrm{SI}$ \\
\hline Covered phase space MD/PELE: ER (1U3S) & $\mathrm{SI}$ \\
\hline LRMSD and backbone RMSD from MD simulations: Syk (4IOS) & $\mathrm{SI}$ \\
\hline Covered phase space MD/PELE: Syk (4IOS) & $\mathrm{SI}$ \\
\hline LRMSD and backbone RMSD from MD simulations: Syk (4XR8) & $\mathrm{SI9}$ \\
\hline Covered phase space MD/PELE: Syk (4XR8) & $\mathrm{SI9}$ \\
\hline LRMSD and backbone RMSD from MD simulations: Abl (2HYY) & $\mathrm{SI10}$ \\
\hline Covered phase space MD/PELE: Abl (2HYY) & $\mathrm{SI10}$ \\
\hline LRMSD and backbone RMSD from MD simulations: Abl (2F4J) & $\mathrm{SI11}$ \\
\hline Covered phase space MD/PELE: Abl (2F4J) & $\mathrm{SI11}$ \\
\hline LRMSD and backbone RMSD from MD simulations: c-Src (1Y57) & $\mathrm{SI12}$ \\
\hline Covered phase space MD/PELE: c-Src (1Y57) & $\mathrm{SI12}$ \\
\hline LRMSD and backbone RMSD from MD simulations: c-Src (1YOM) & $\mathrm{SI13}$ \\
\hline Covered phase space MD/PELE: c-Src (1YOM) & $\mathrm{SI13}$ \\
\hline
\end{tabular}


A) c-Src (1Y57)

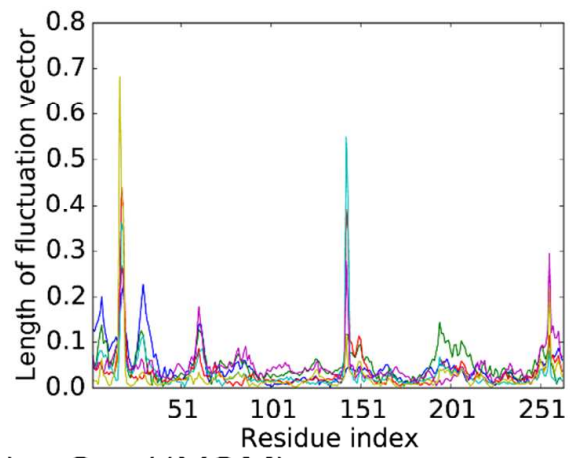

B) C-Src (1YOM)

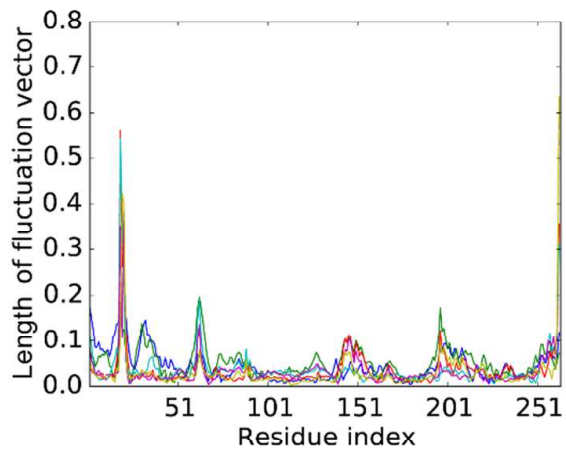

C) Abl (2F4J)

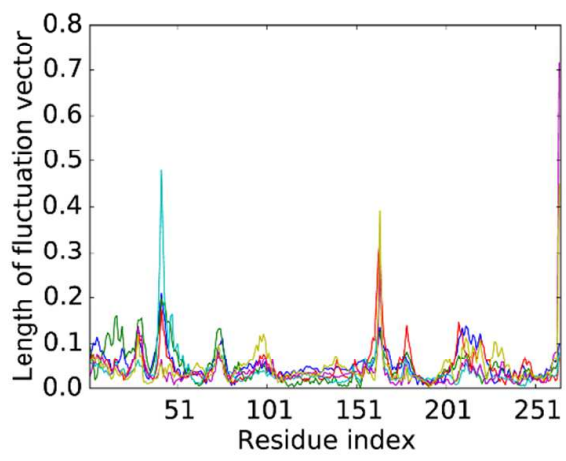

D) $\mathrm{Abl}(2 \mathrm{HYY})$

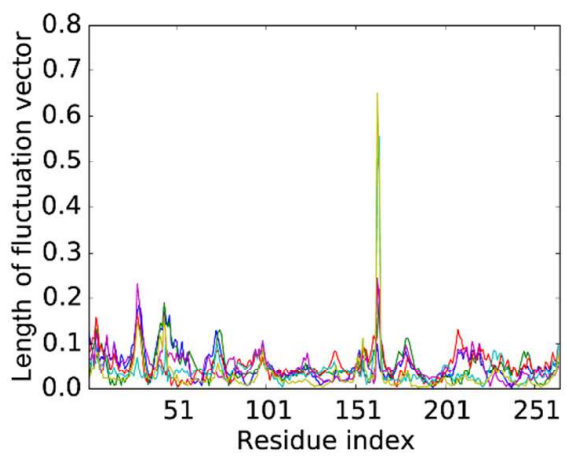

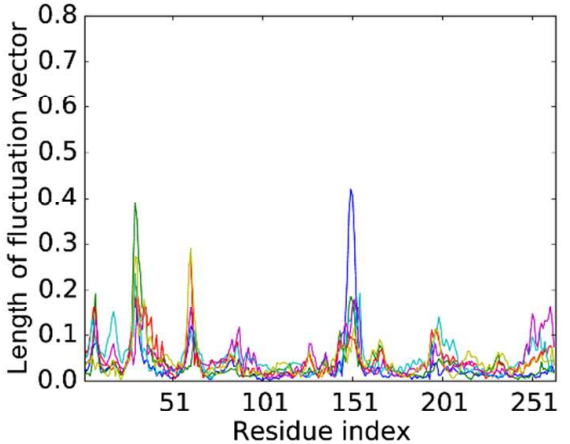
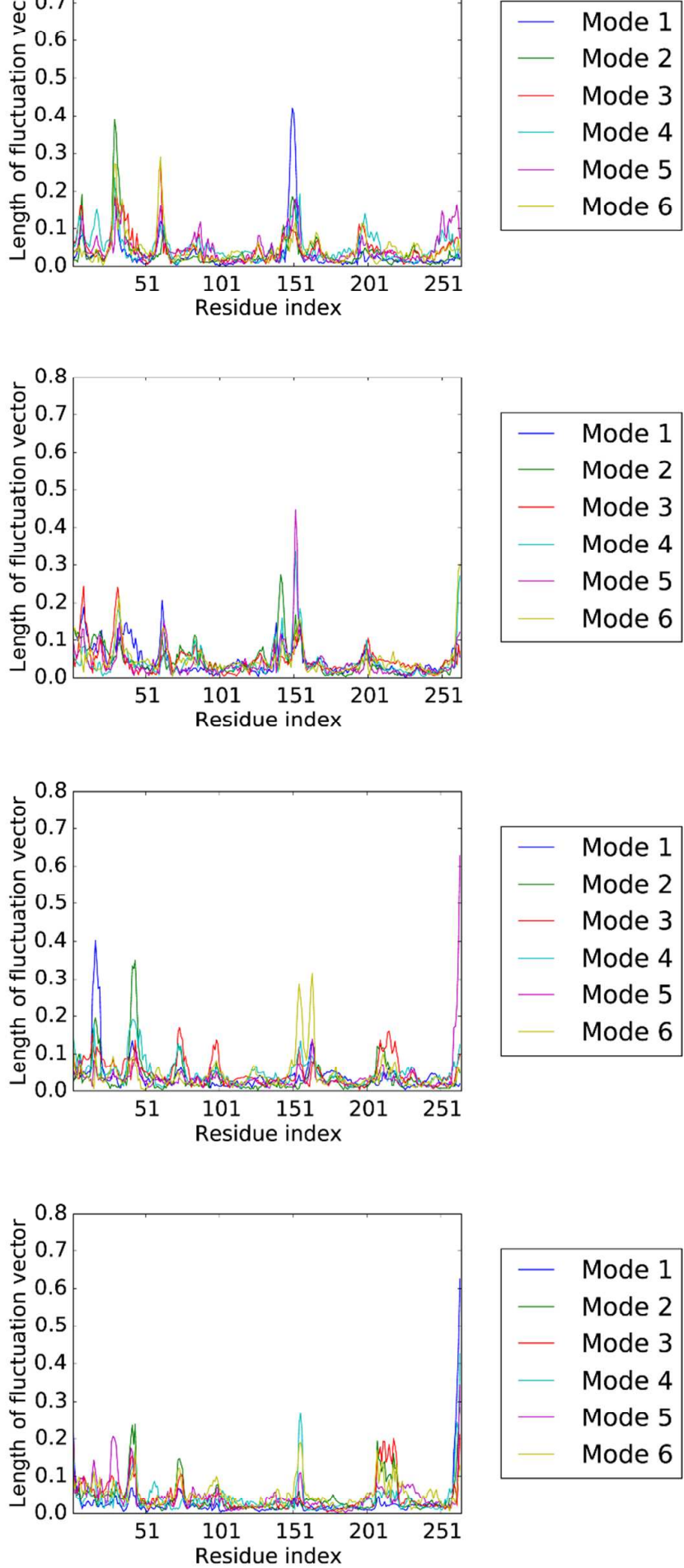

Figure S1 First six normal modes from ANM and the first six principal components from PCA of the MD simulations for c-Src and Abl kinase. 

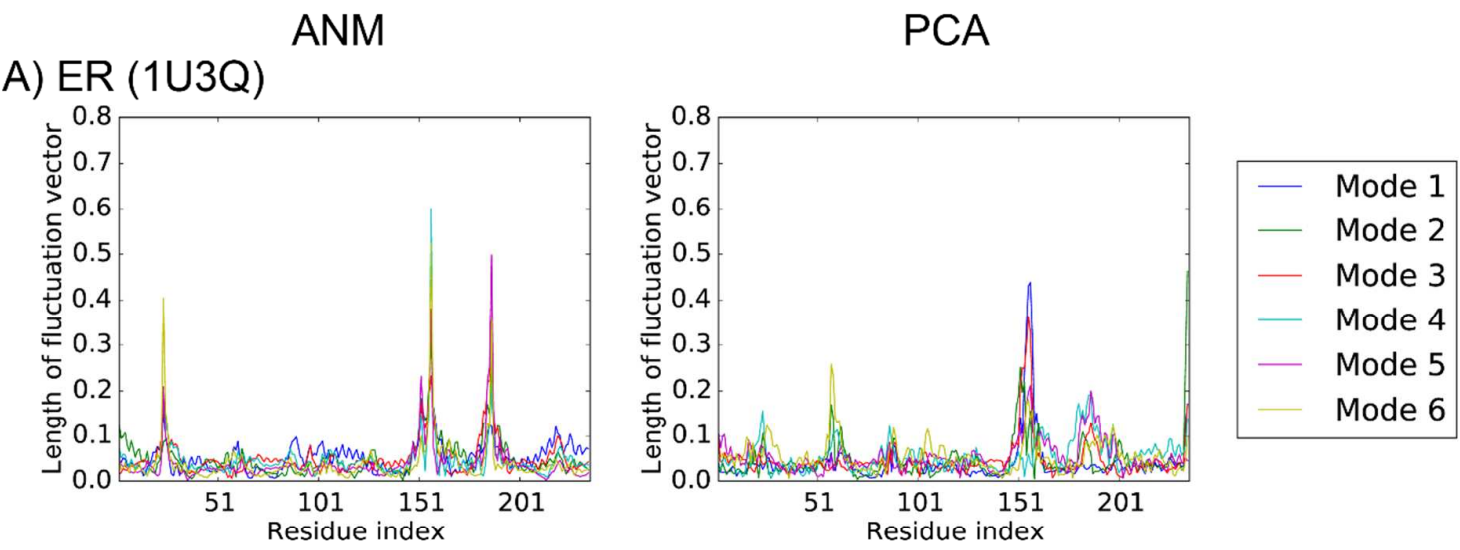

\section{B) ER (1U3S)}
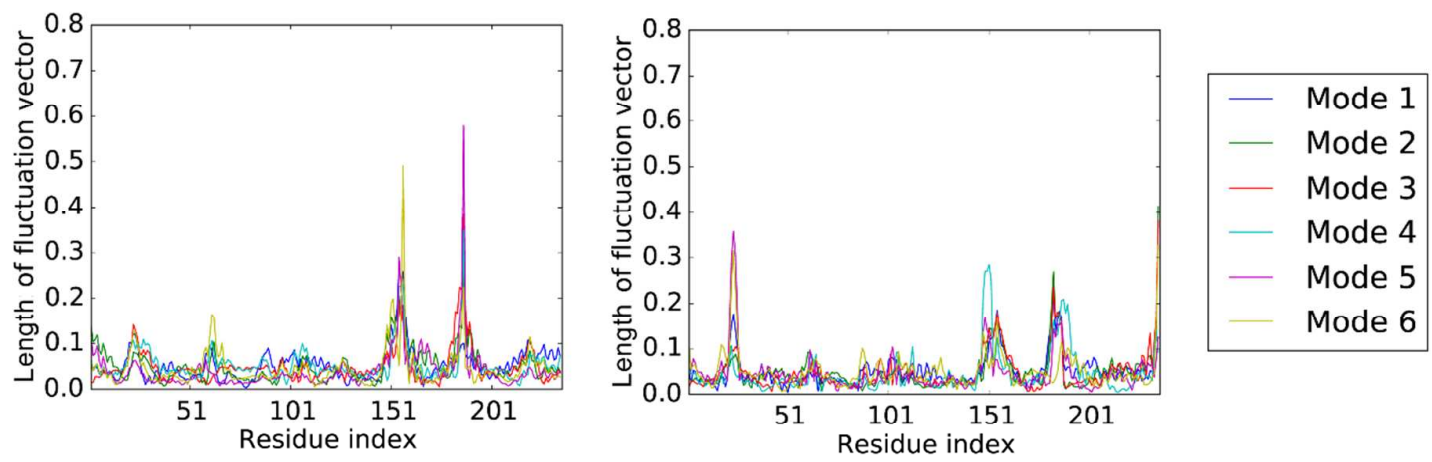

\section{C) HNE (3Q76)}
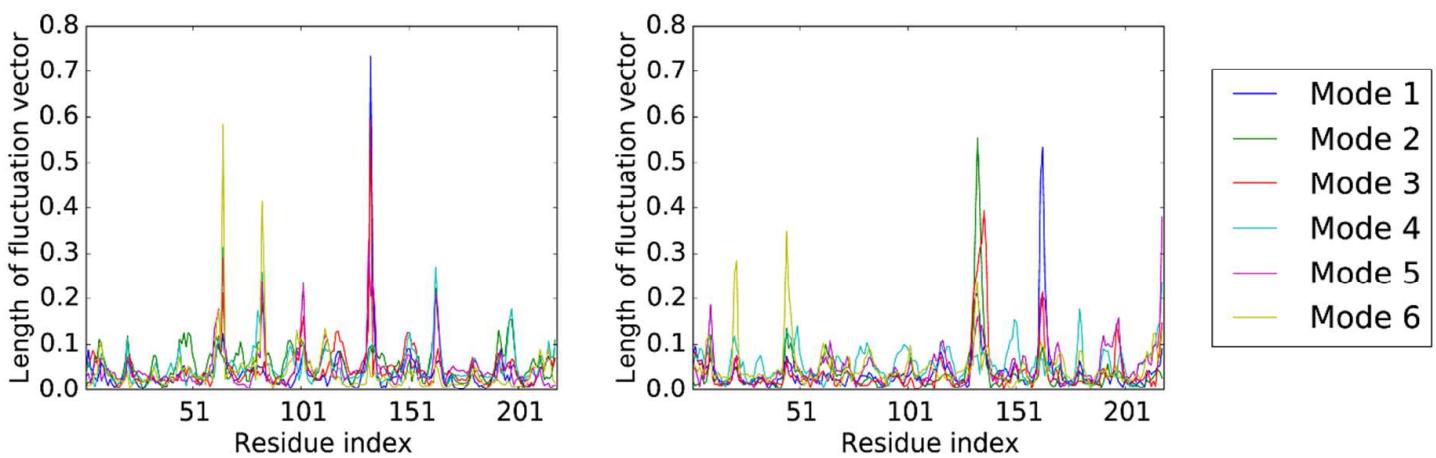

Figure S2 First six normal modes from ANM and the first six principal components from PCA of the MD simulations for ER and HNE. 
ANM

PCA

A) SYK (4IOS)
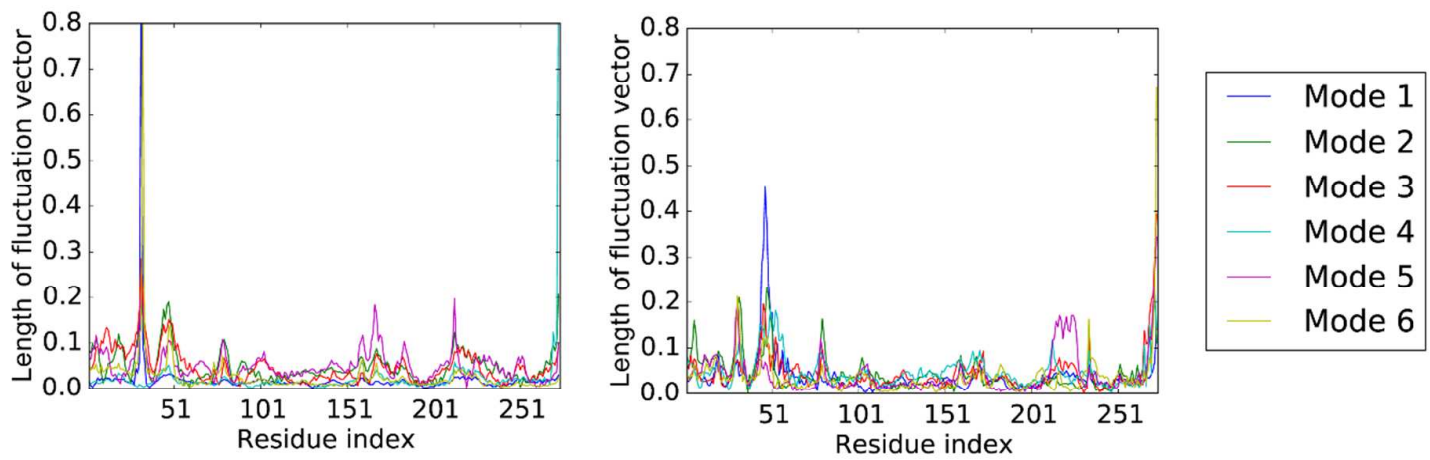

B) SYK (4XR8)
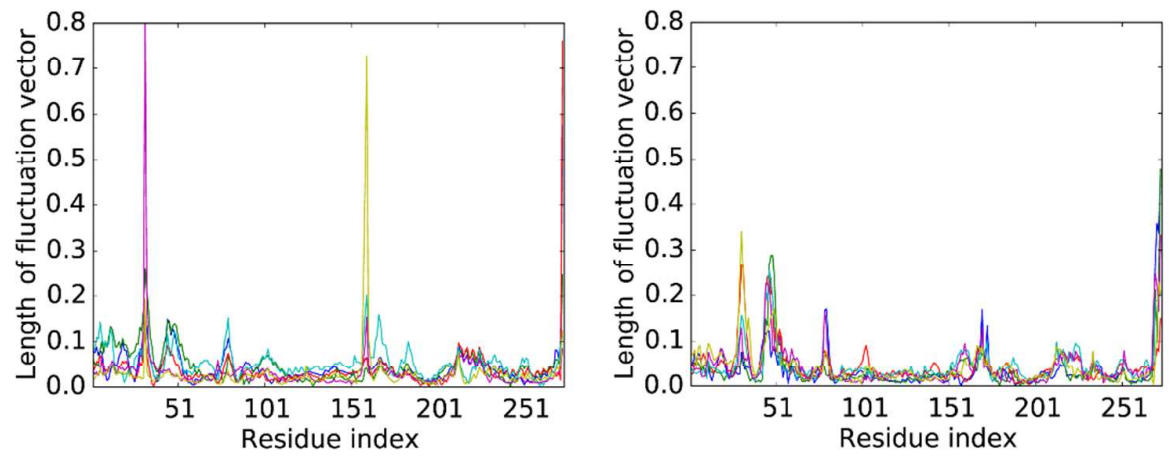

$-\quad$ Mode 1
$-\quad$ Mode 2
$-\quad$ Mode 3
$-\quad$ Mode 4
$-\quad$ Mode 5
$-\quad$ Mode 6

Figure S3 First six normal modes from ANM and the first six principal components from PCA of the MD simulations for the SYK kinase. 


\section{HNE}
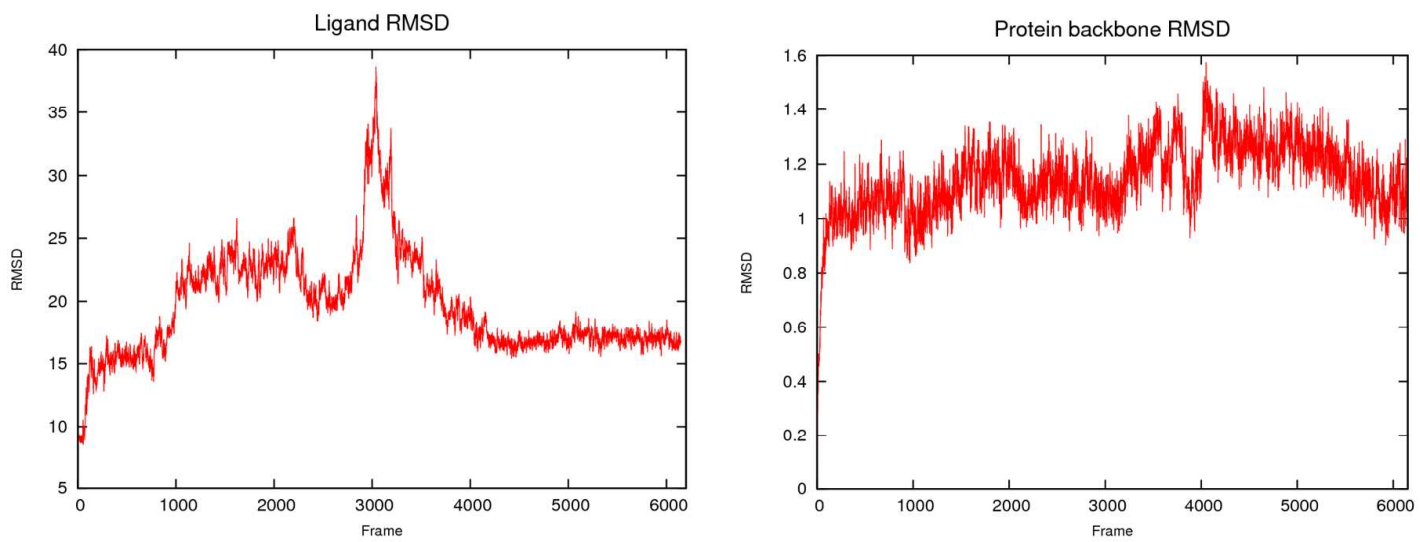

Figure S4 LRMSD and backbone RMSD of $3 Q 77$ during MD simulation.
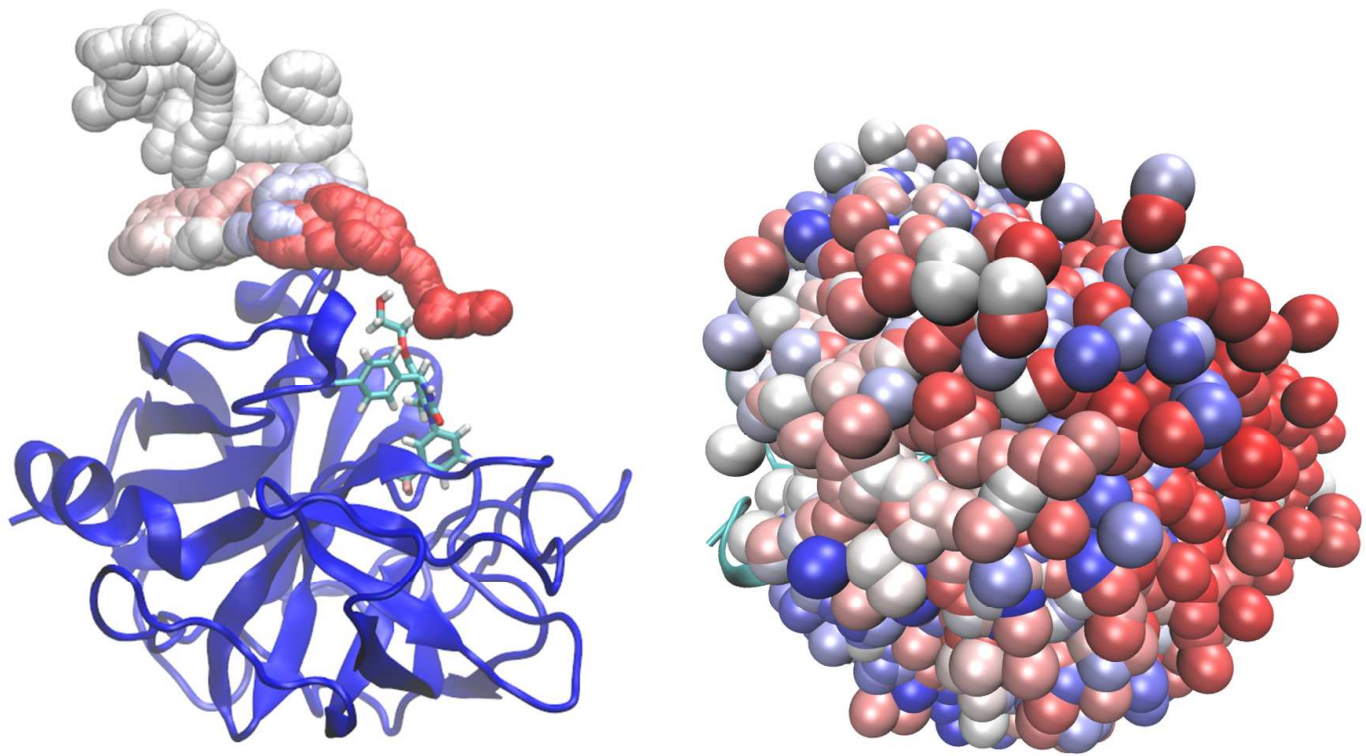

Figure S5 Ligand positions during the MD (left) and the PELE (right) simulation for 3Q77. The central atom of the ligand is indicated by a sphere colored due to its simulation step (red to blue). 

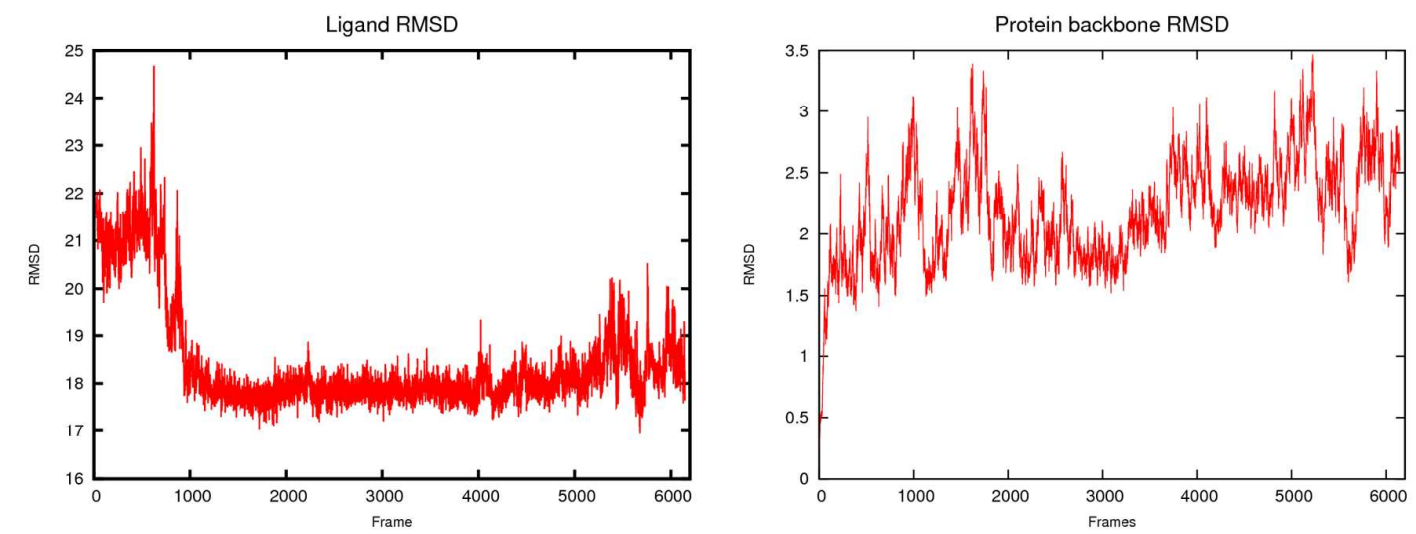

Figure S6 LRMSD and backbone RMSD of $1 \mathrm{U} 3 \mathrm{Q}$ during MD simulation.
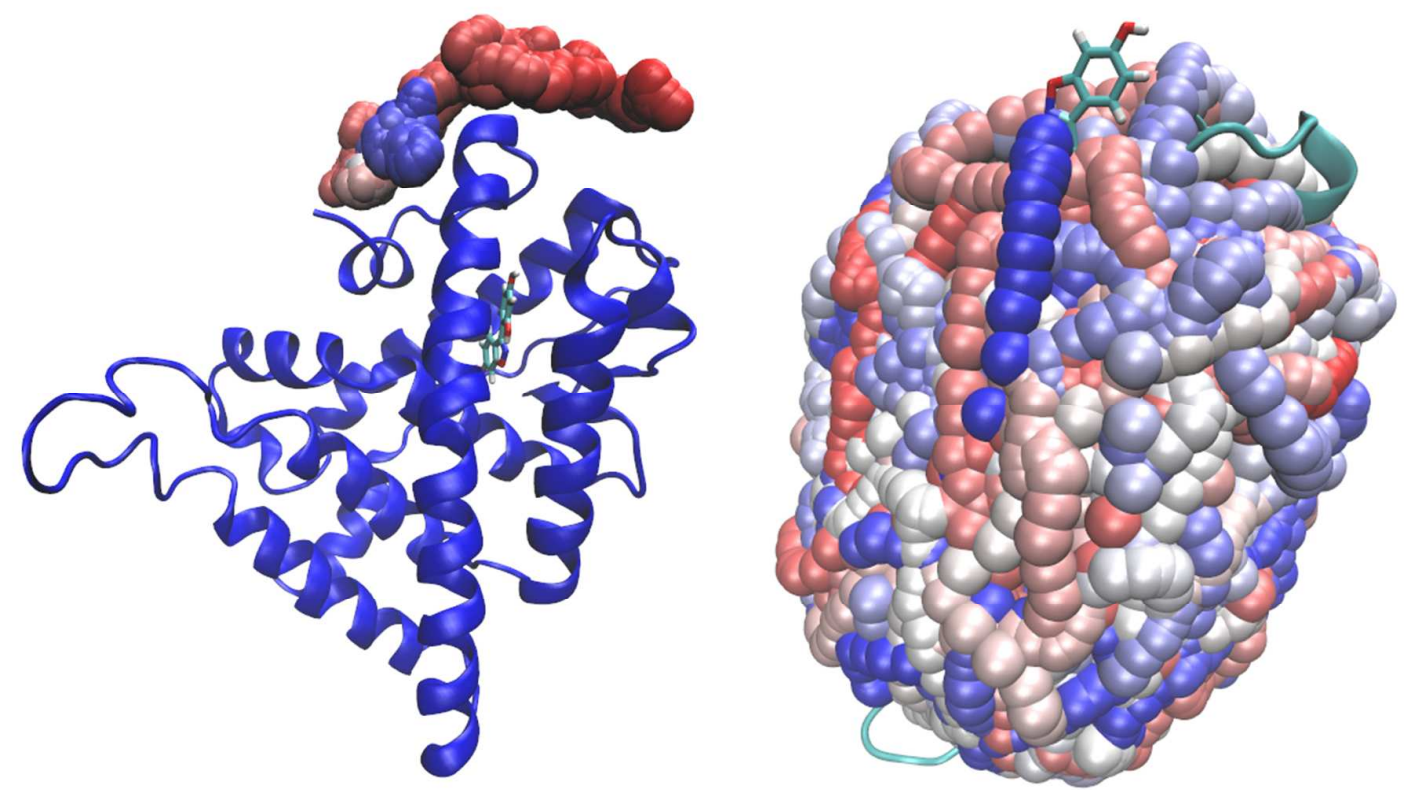

Figure S7 Ligand positions during the MD (left) and the PELE (right) simulation for $1 \mathrm{U} 3 \mathrm{Q}$. The central atom of the ligand is indicated by a sphere colored due to its simulation step (red to blue). 

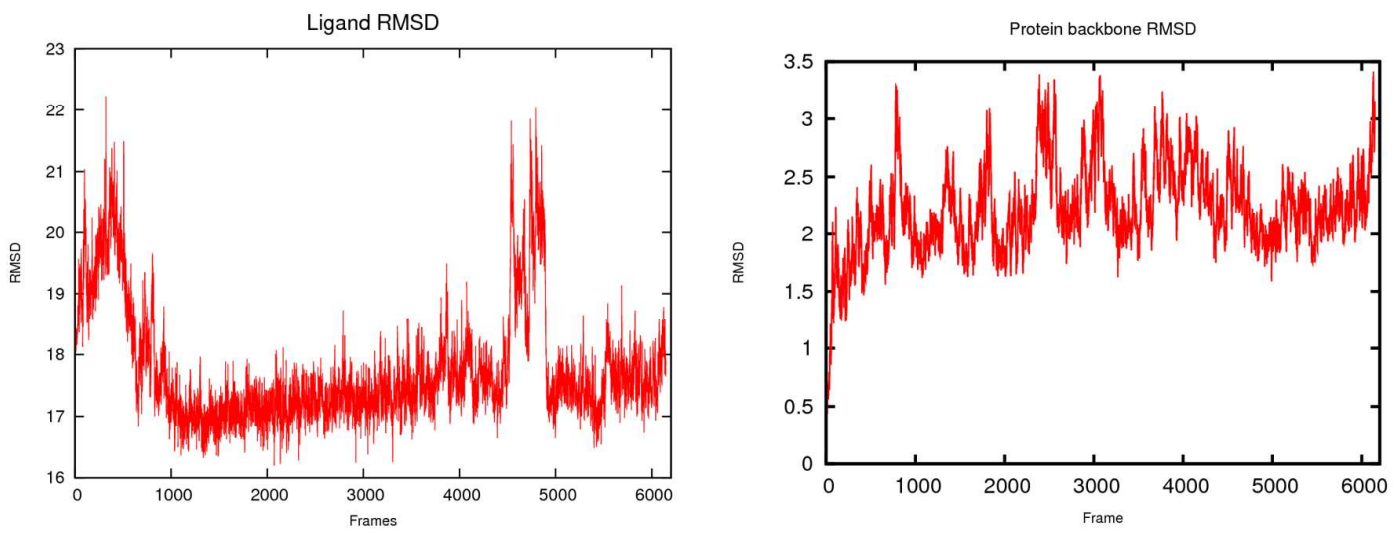

Figure S8 LRMSD and backbone RMSD of 1U3S during MD simulation.
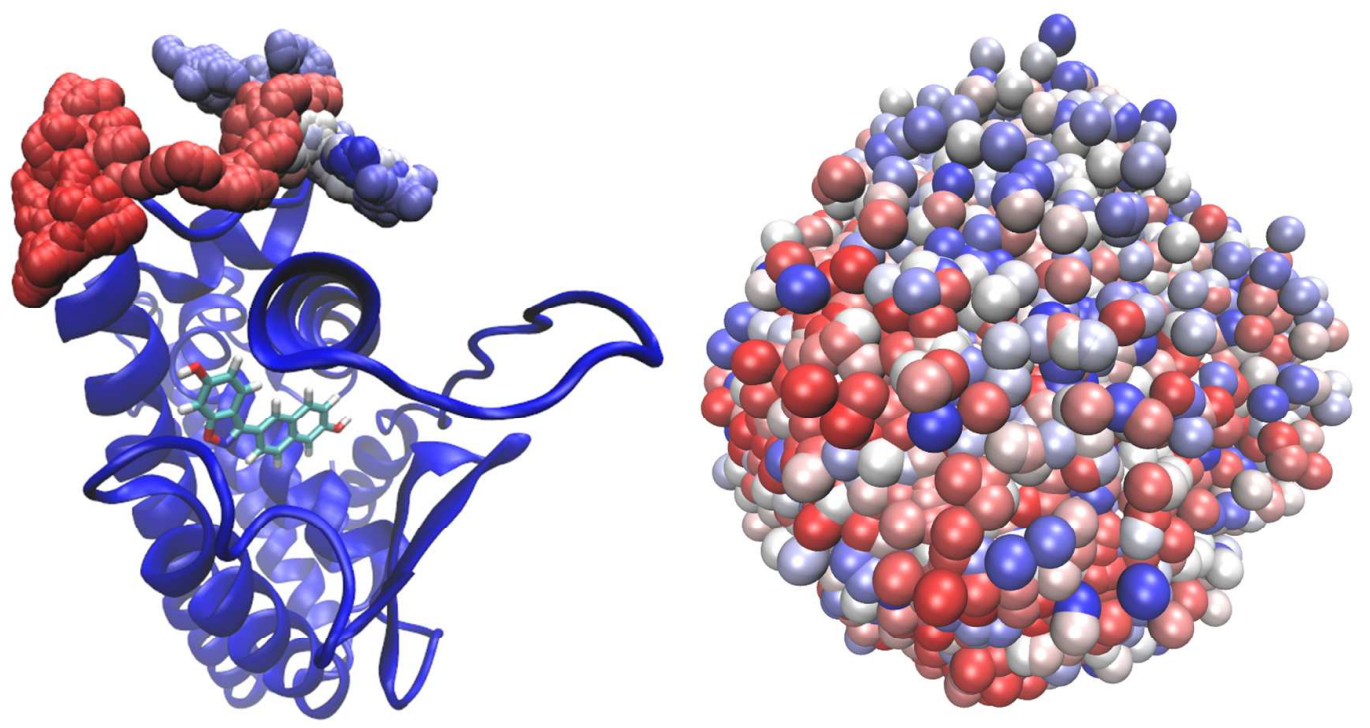

Figure S9 Ligand positions during the MD (left) and the PELE (right) simulation for 1U3S. The central atom of the ligand is indicated by a sphere colored due to its simulation step (red to blue). 


\section{$\underline{S Y K}$}

$4 I O S$
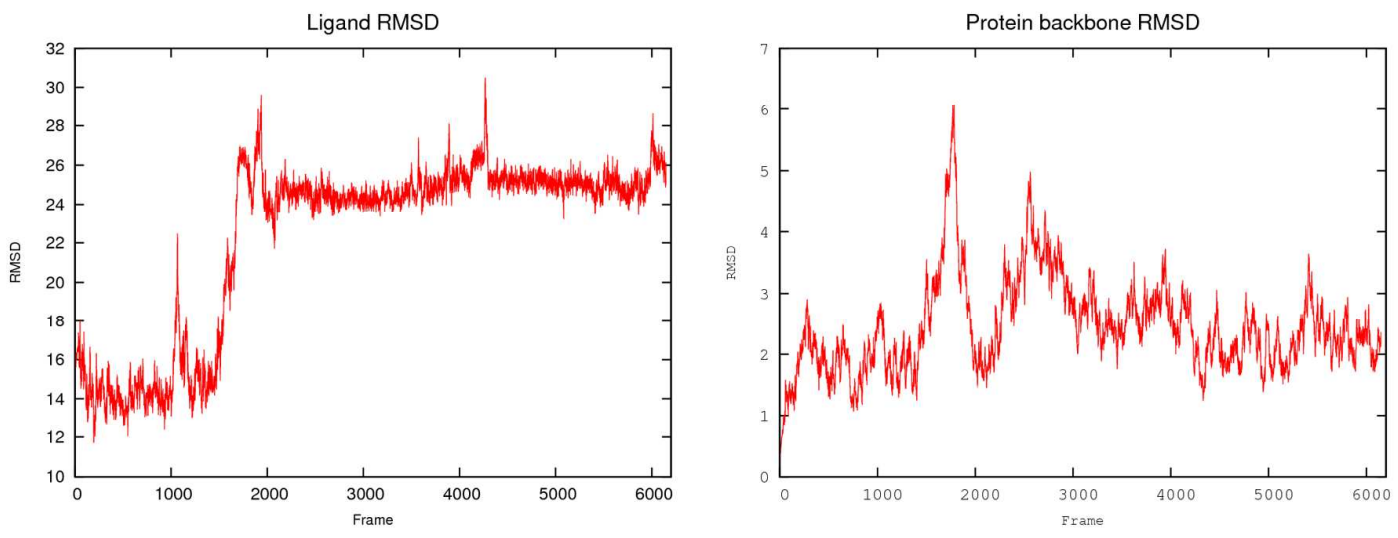

Figure S10 LRMSD and backbone RMSD of 4IOS during MD simulation.
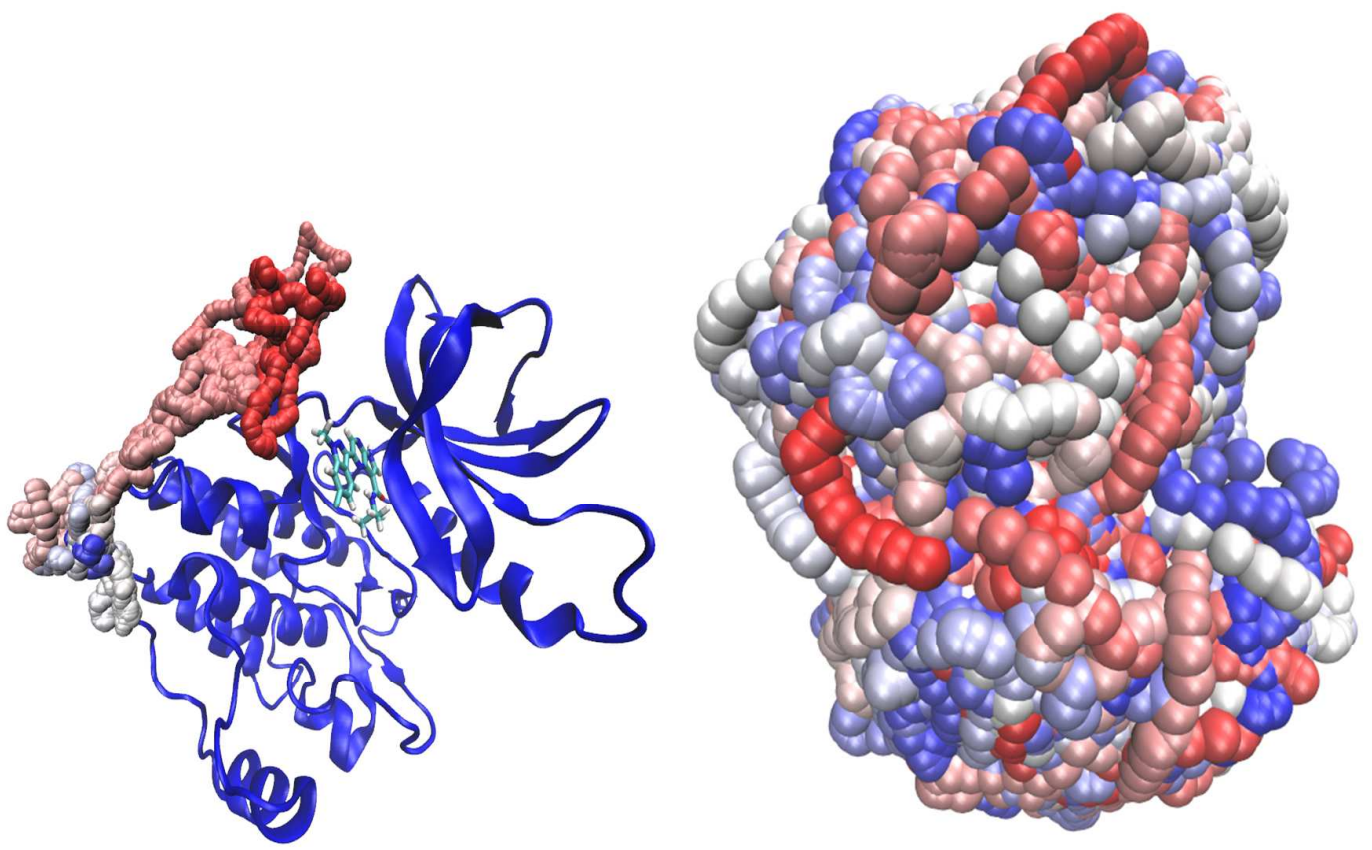

Figure S11 Ligand positions during the MD (left) and the PELE (right) simulation for 4IOS. The central atom of the ligand is indicated by a sphere colored due to its simulation step (red to blue). 


\section{XR8}
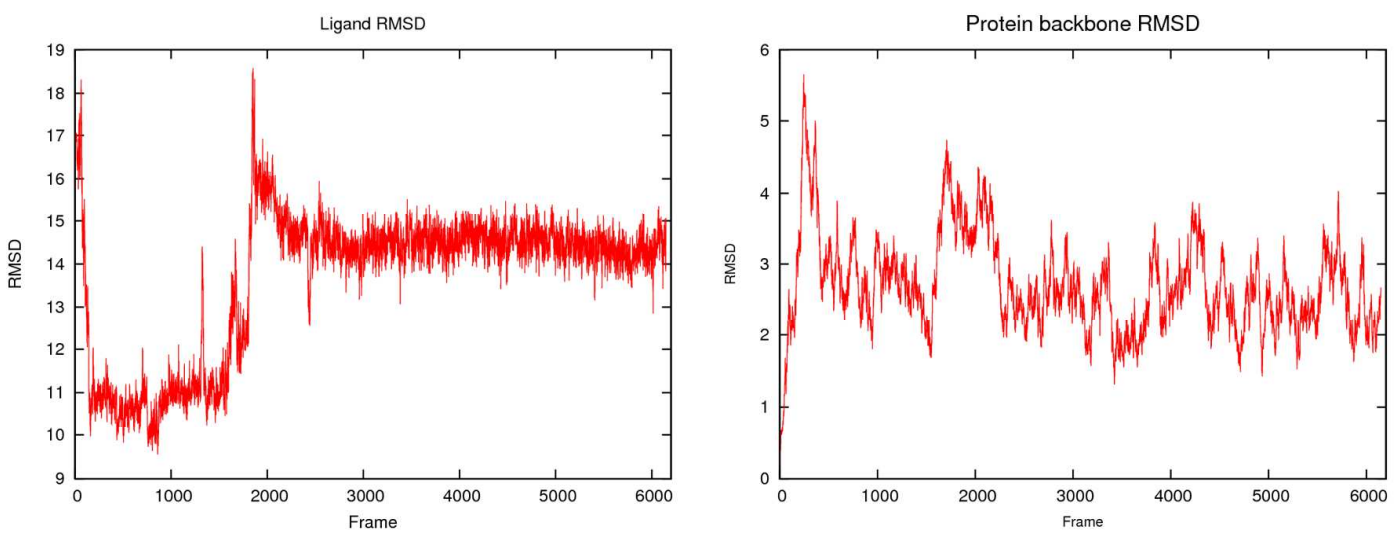

Figure S12 LRMSD and backbone RMSD of 4XR8 during MD simulation.
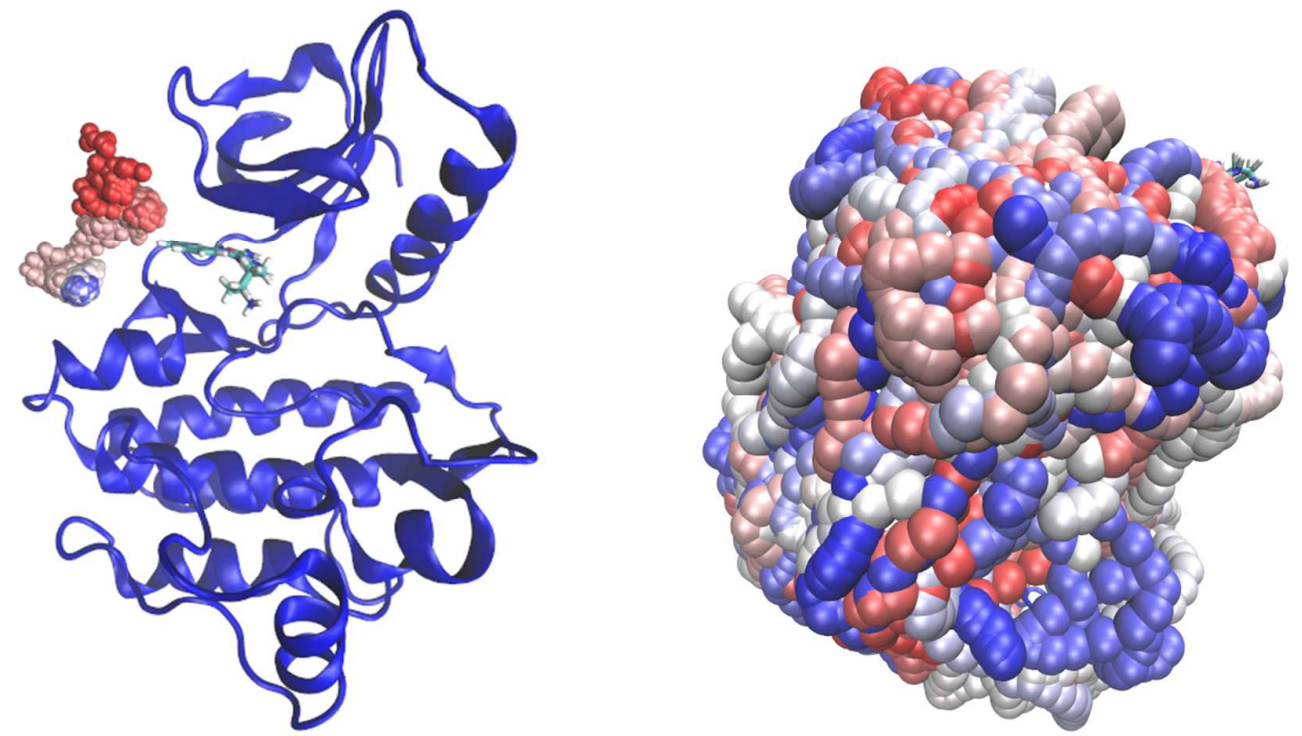

Figure S13 Ligand positions during the MD (left) and the PELE (right) simulation for 4XR8. The central atom of the ligand is indicated by a sphere colored due to its simulation step (red to blue). 


\section{$\underline{A b l}$}

2HYY
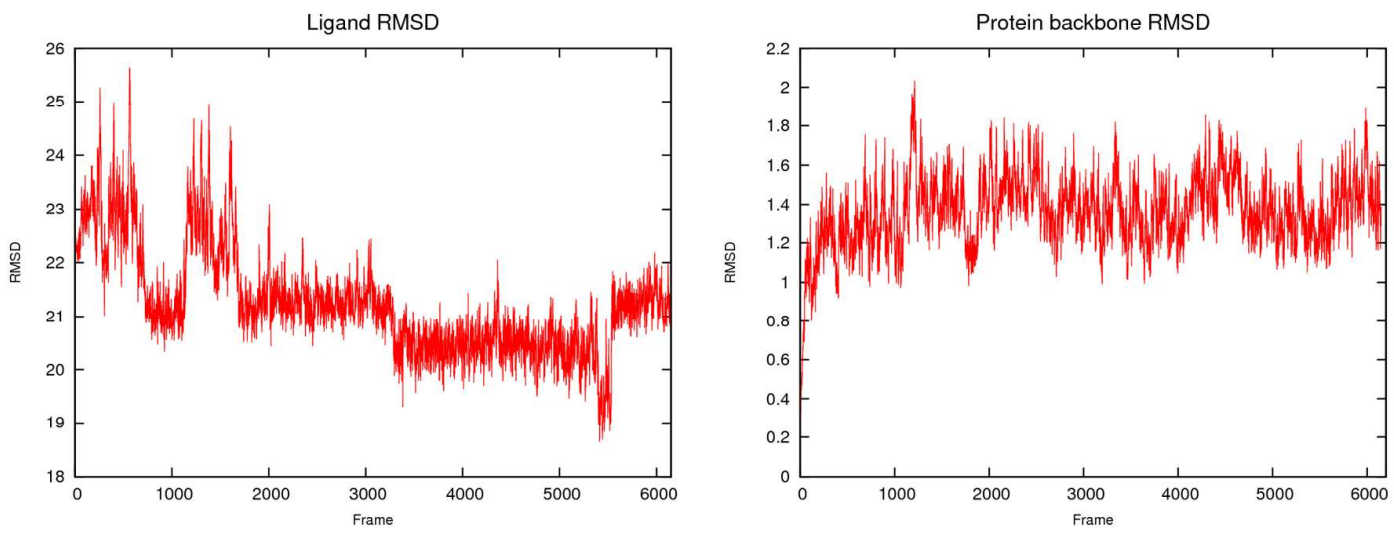

Figure S14 LRMSD and backbone RMSD of 2HYY during MD simulation.
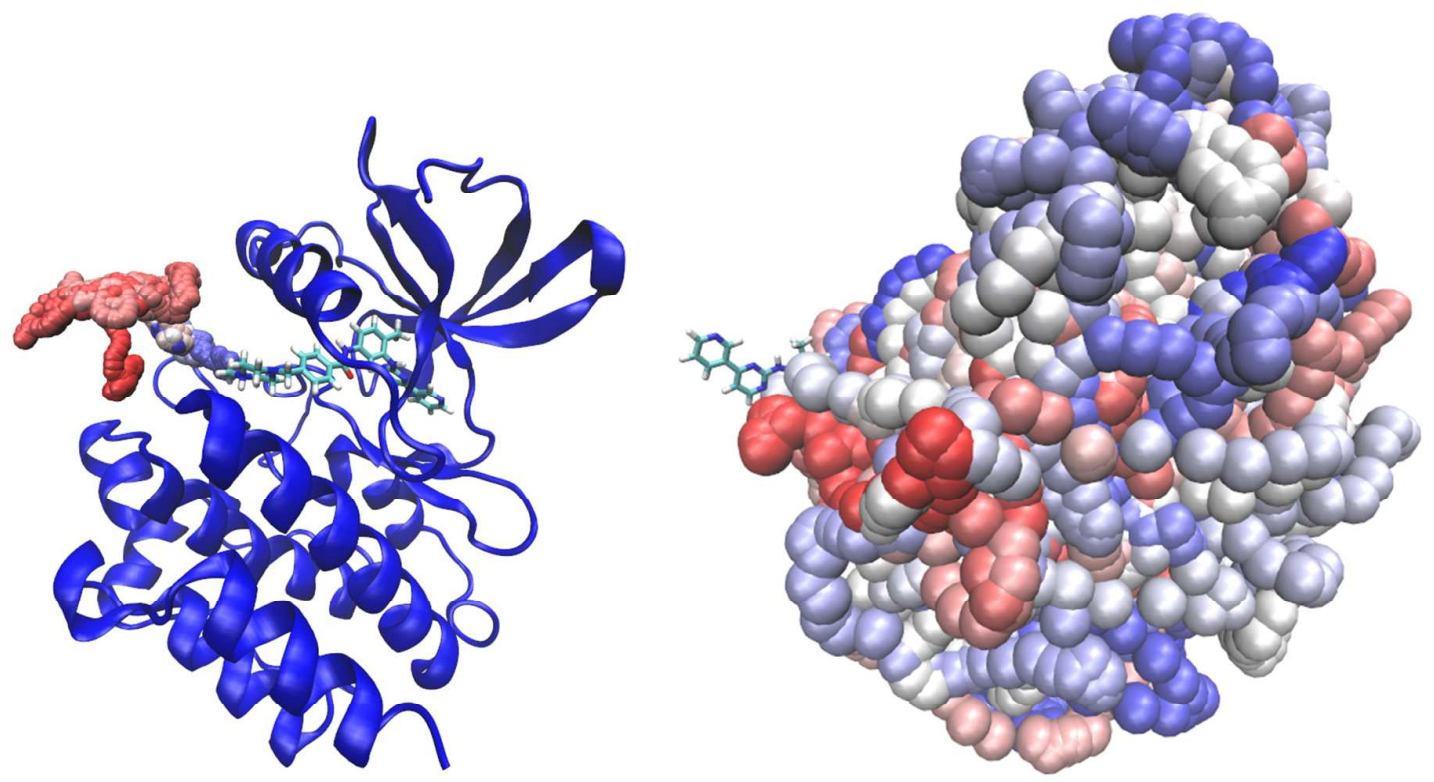

Figure S15 Ligand positions during the MD (left) and the PELE (right) simulation for 2HYY. The central atom of the ligand is indicated by a sphere colored due to its simulation step (red to blue). 


\section{F4J}
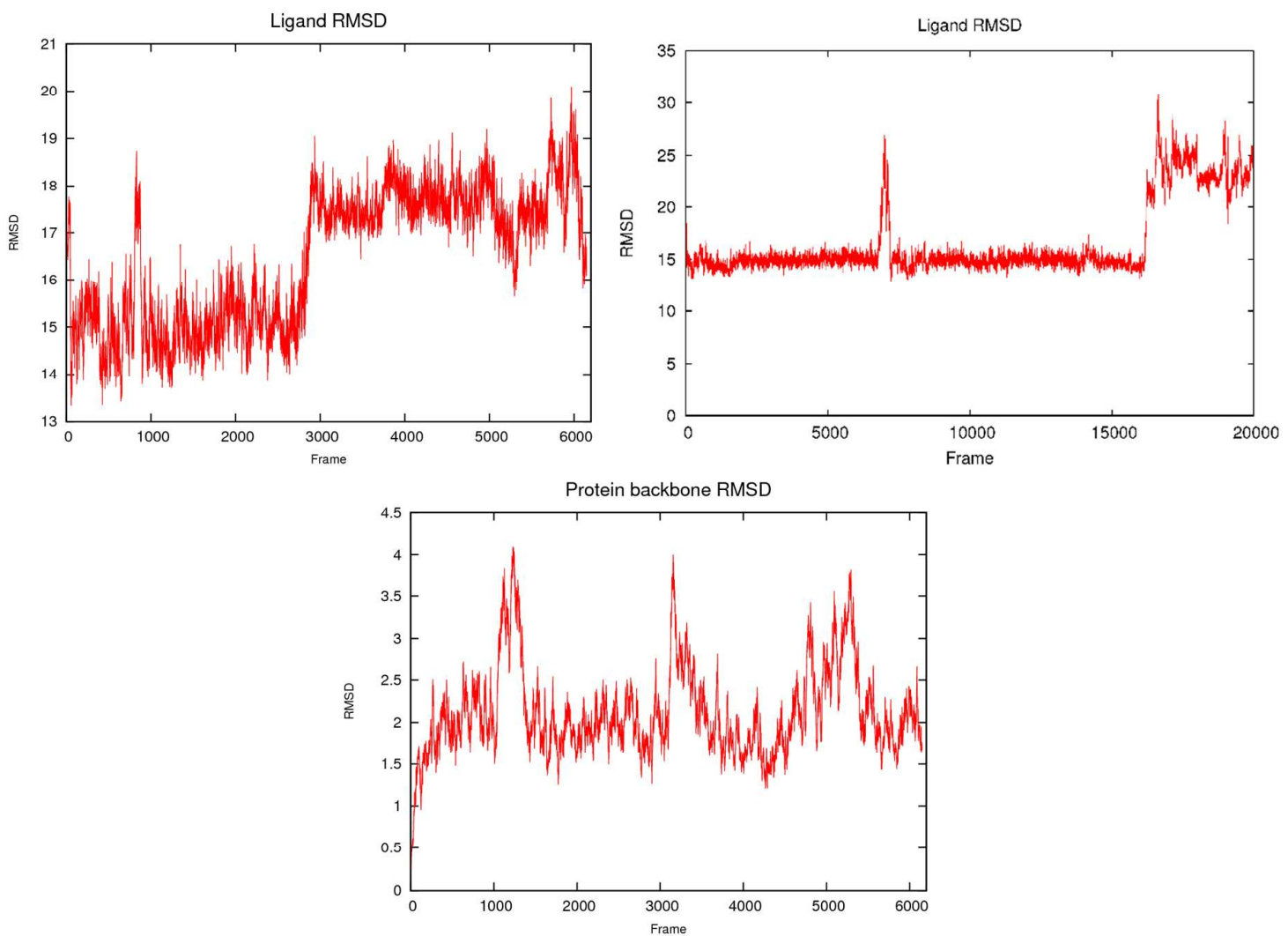

Figure S16 LRMSD (for 60ns and 200ns) and backbone RMSD of 2F4J during MD simulation.
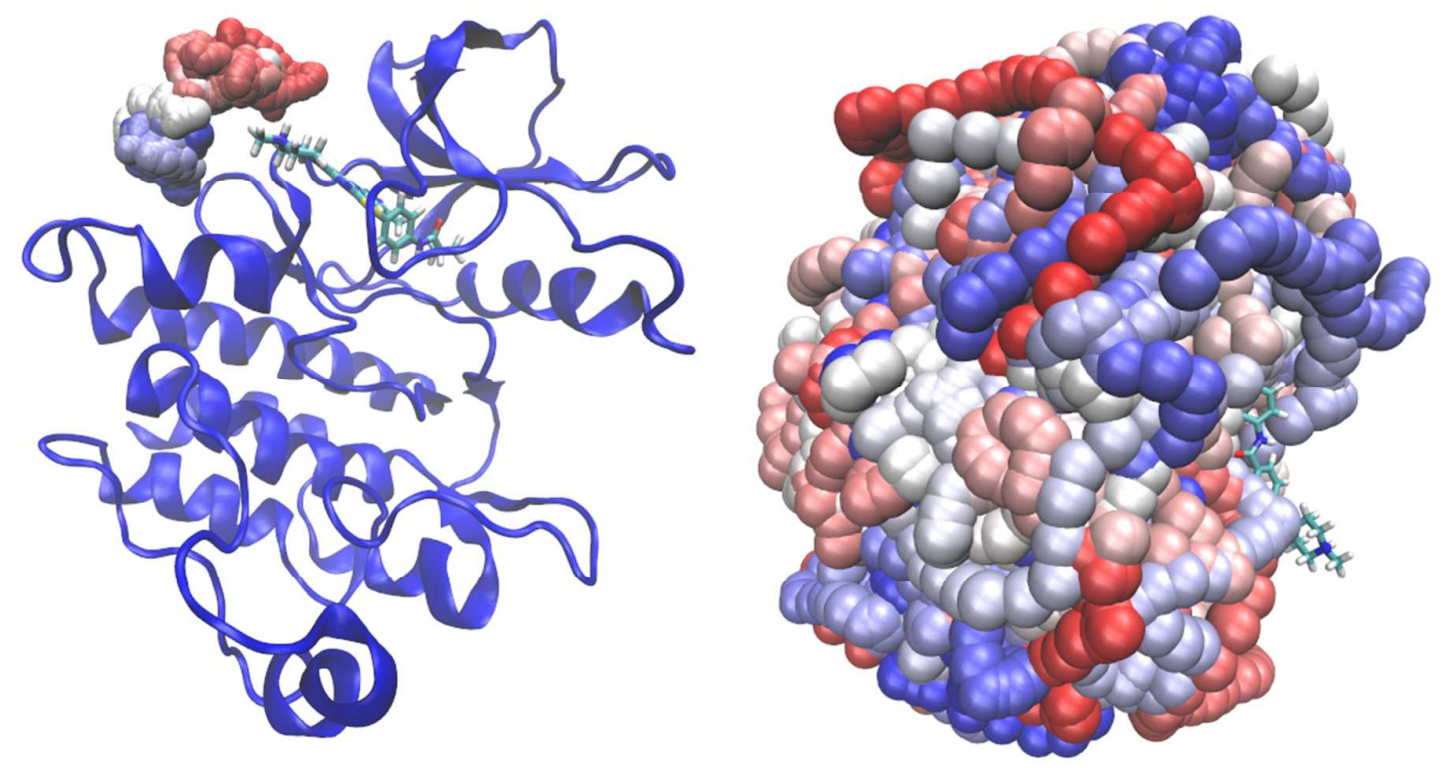

Figure S17 Ligand positions during the MD (left) and the PELE (right) simulation for 2F4J. The central atom of the ligand is indicated by a sphere colored due to its simulation step (red to blue). 

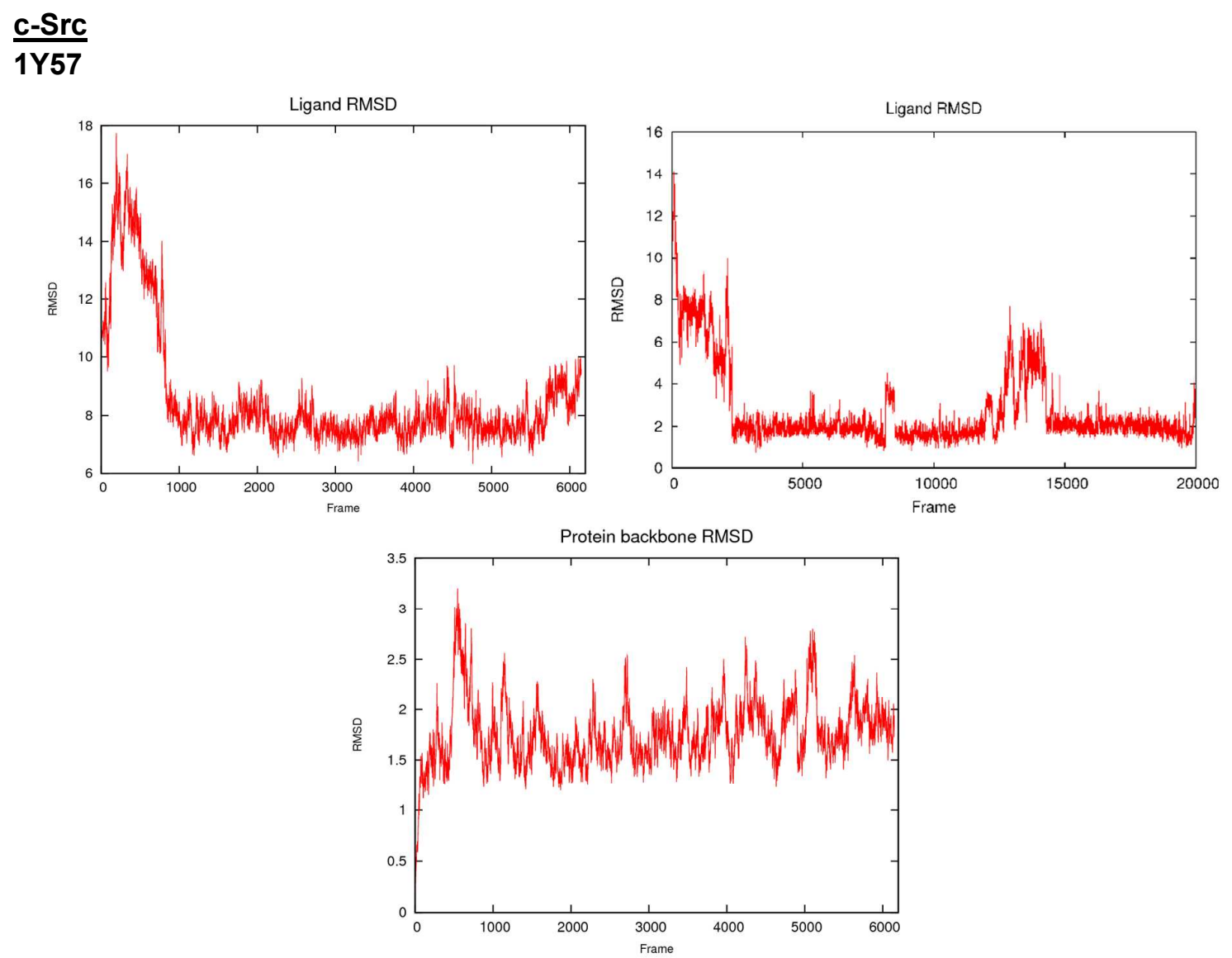

Figure S18 LRMSD (for 60ns and 200ns) and backbone RMSD of 1 Y57 during MD simulation.
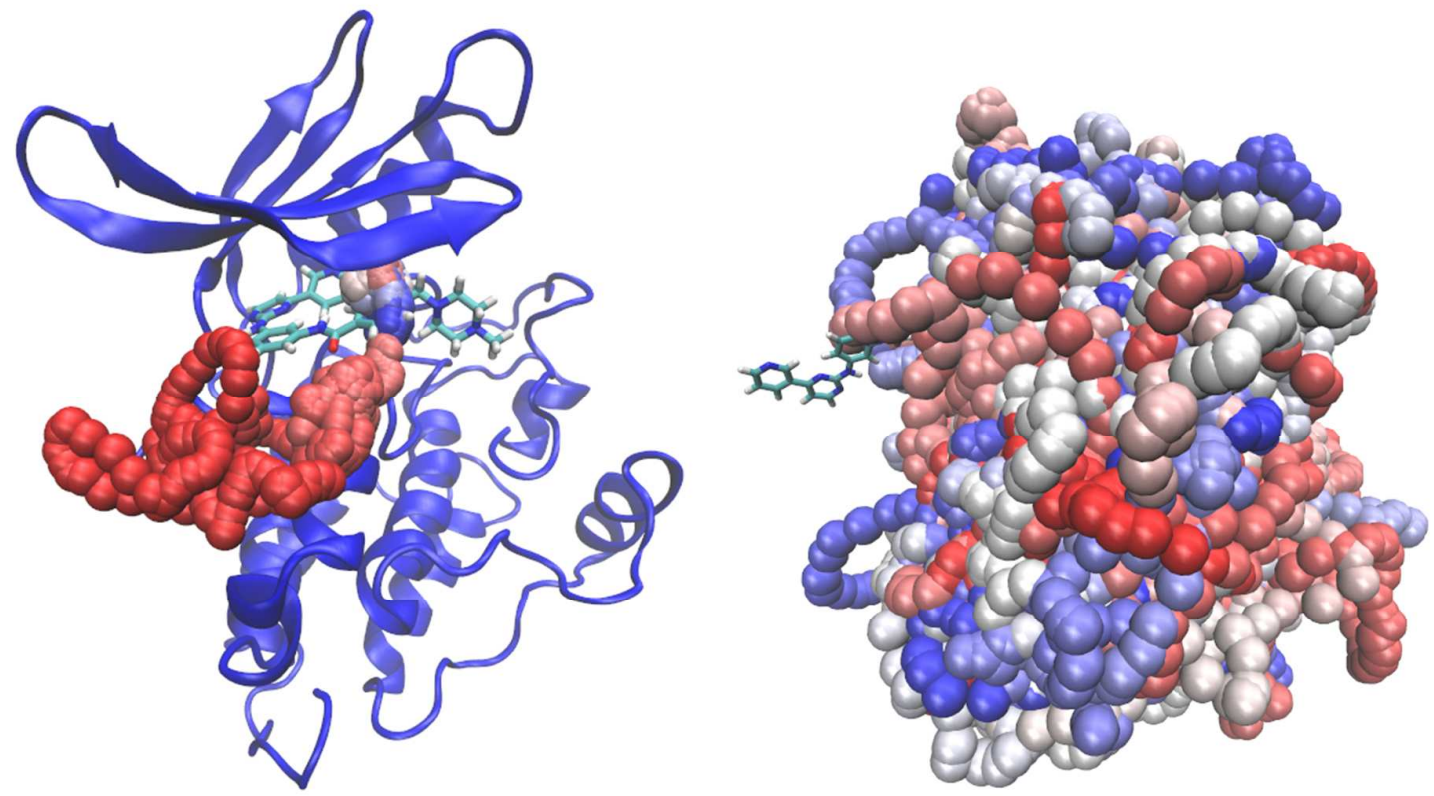

Figure S19 Ligand positions during the MD (left) and the PELE (right) simulation for 1 Y57. The central atom of the ligand is indicated by a sphere colored due to its simulation step (red to blue). 
1YOM
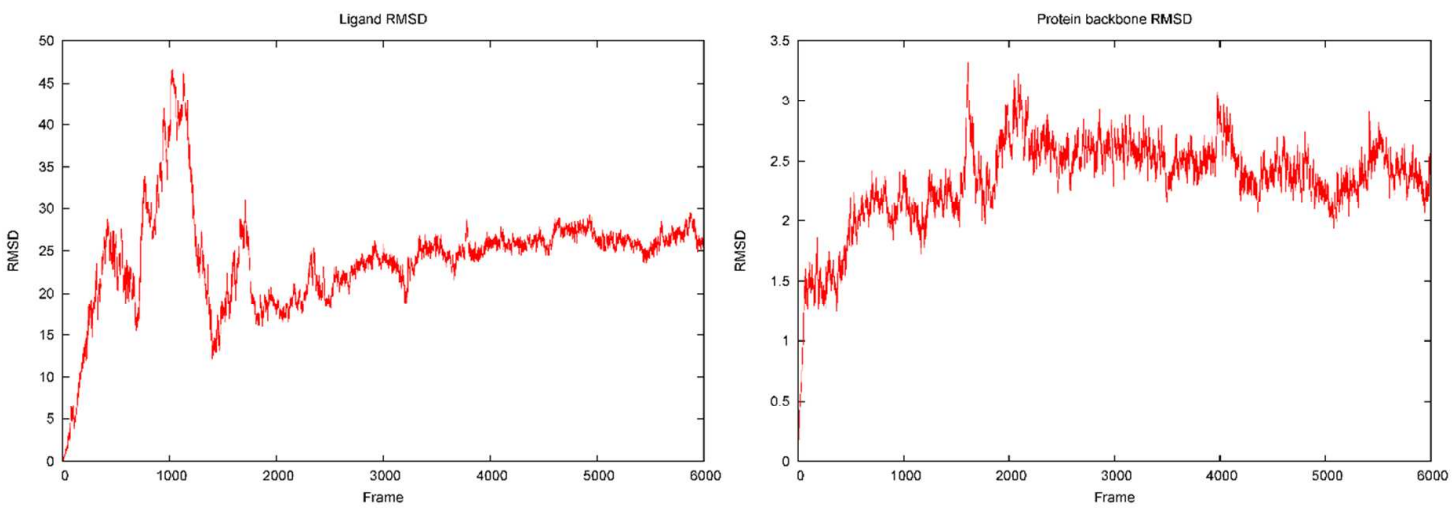

Figure S20 LRMSD and backbone RMSD of 1YOM during MD simulation.
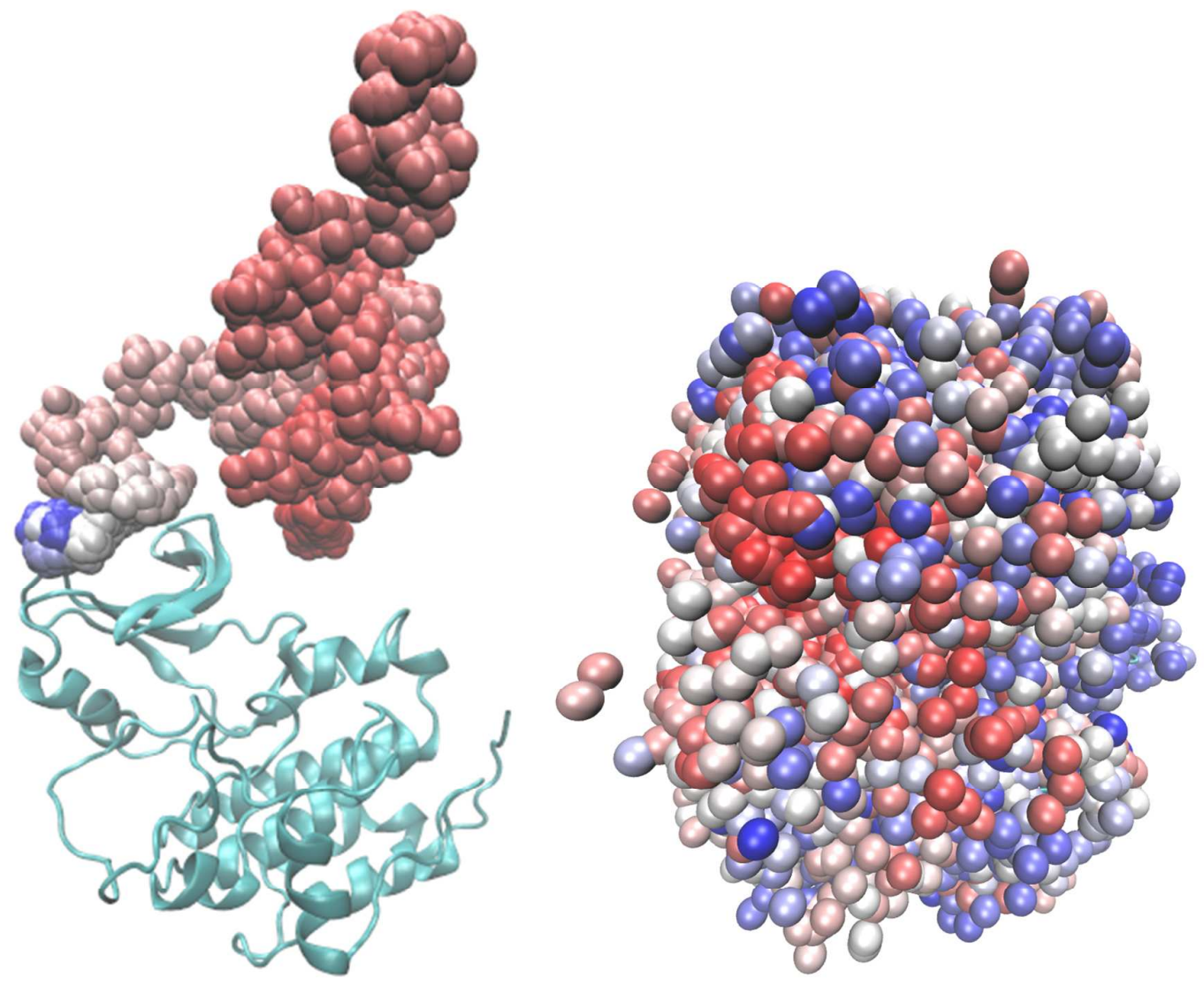

Figure S21 Ligand positions during the MD (left) and the PELE (right) simulation for 1YOM. The central atom of the ligand is indicated by a sphere colored due to its simulation step (red to blue). 